Revue de l'Institut des langues et cultures

d'Europe, Amérique, Afrique, Asie et Australie

$32 \mid 2018$

Didactique des langues et cultures de spécialité : méthodes, corpus et nouvelles technologies

\title{
Le français sur objectif spécifique : la notion d' authentique revisitée
}

French for Specific Purposes: Revisiting Authenticity

Sophie Dufour et Chantal Parpette

\section{OpenEdition}

\section{Journals}

Édition électronique

URL : http://journals.openedition.org/ilcea/4814

DOI : 10.4000/ilcea.4814

ISSN : 2101-0609

Éditeur

UGA Éditions/Université Grenoble Alpes

Édition imprimée

ISBN : 978-2-37747-059-4

ISSN : 1639-6073

Référence électronique

Sophie Dufour et Chantal Parpette, « Le français sur objectif spécifique : la notion d'authentique revisitée », ILCEA [En ligne], 32 | 2018, mis en ligne le 01 juillet 2018, consulté le 30 avril 2019. URL:

http://journals.openedition.org/ilcea/4814; DOI : 10.4000/ilcea.4814

Ce document a été généré automatiquement le 30 avril 2019

(C) ILCEA 


\section{Le français sur objectif spécifique : la notion d'authentique revisitée}

French for Specific Purposes: Revisiting Authenticity

Sophie Dufour et Chantal Parpette

\section{Introduction}

1 Les programmes de langue sur objectif spécifique constituent un domaine privilégié des documents authentiques. En effet, leur élaboration suppose pour le concepteur d'explorer un contexte qui ne lui est pas familier, et de partir à la découverte des situations, des acteurs, et des discours qui le constituent. Cela explique le rôle fondamental du travail de terrain dans la construction de ces programmes, et la place centrale de la collecte des données dans la démarche décrite par Mangiante et Parpette (2004: 46-55) pour le français sur objectif spécifique (désormais FOS). Cette prospection in situ a pour corollaire la présence importante de données authentiques dans les formations de langues sur objectif spécifique, que nous analyserons ici à travers l'exemple particulier du FOS. Pourquoi se pencher aujourd'hui sur une notion qui a émergé il y a plus de quarante ans en didactique des langues et génère depuis longtemps des outils d'enseignement ? Parce que la notion d'authentique, qui fait globalement consensus, s'accompagne depuis son origine d'interrogations qui, tout en ayant trouvé au fil du temps un certain nombre de réponses méthodologiques, suscite encore des réflexions et des choix de conception. Nous analysons ici la relation que les discours authentiques, notamment oraux, entretiennent avec leur contexte d'origine, l'impact de leur transfert dans le contexte des formations linguistiques, et les stratégies nées dans le champ du FOS pour créer une nouvelle approche des situations et des données authentiques. 


\section{Petite histoire des documents authentiques}

2 Dans les années 1970 émerge une réflexion critique sur les méthodes structuralistes d'enseignement du FLE, que Boyer, Butzbach et Pendanx formulent ainsi :

Un sentiment a progressivement prévalu au sein de la didactique duFLE: le sentiment que les dialogues fabriqués de telle ou telle méthode présentaient une langue peu naturelle et qu'ils étaient par top éloignés de l'authentique communication entre Français. La pseudo-parole proposée souvent dans les manuels semblait méconnaître aussi bien les ressorts de l'affectivité que la variation socioculturelle. D'où un plaidoyer de plus en plus répandu pour "l'authentique » et plus particulièrement pour une attention conséquente à la diversité des «façons » de parler et des situations de communication. (1990:27)

Cette réflexion ouvre la voie à l'évolution méthodologique qui aboutira à l'approche communicative. Celle-ci, adossée à des concepts de la pragmatique, notamment ceux de situation de communication, contexte, énonciation, se tourne davantage vers la dimension socioculturelle de la communication et les discours en situation, et tente de prendre en charge les variations langagières liées aux différents profils des locuteurs et aux divers enjeux de leurs échanges. On voit ainsi apparaitre dans les méthodes C'est le printemps (Montredon et al., 1976), puis Archipel (Courtillon \& Raillard, 1988) et d'autres ensuite, des profils de locuteurs et des modes de communication visant à représenter davantage la diversité présente dans la société française. Cela fait émerger la notion d'authenticité qui va se manifester dans différents aspects des situations langagières traitées : les profils des locuteurs mis en scène (cadre, étudiant, logeuse, travailleur immigré, artiste), leurs caractères (anticonformiste, sérieux, dragueur, acariâtre), les modes d'expression (explication posée, plaisanterie, préjugés, protestation, critique implicite). Cela apparaît aussi dans les outils linguistiques, dans le vocabulaire certes (différents niveaux de langue, apocopes), mais également dans les enchaînements d'énoncés (« Il habite à Paris ? Ça, j'sais pas! » au lieu du format structuraliste «Il habite à Paris? Non, il n'habite pas à Paris »).

4 Le rapprochement avec les situations réelles se traduit également par l'introduction dans les méthodes des «documents authentiques » qui vont devenir l'un des emblèmes de l'approche communicative. Le Dictionnaire de didactique du français langue étrangère et seconde en donne la définition suivante :

La caractérisation d'authentique en didactique des langues est généralement associée à « document » et s'applique à tout message élaboré par des francophones pour des francophones à des fins de communication réelle : elle désigne donc tout ce qui n'est pas conçu à l'origine pour la classe. (Cuq, 2003: 29)

5 Si la définition fait globalement consensus depuis l'émergence du terme (Abe, Carton, Cembalo et Régent évoquaient "tout document produit à des fins autres que l'apprentissage d'une langue seconde" [1979:2]), l'intérêt pour les documents authentiques n'est pas partagé par tous. Un article de Debyser en 1979 souligne que

le $\mathrm{BELC}^{1}$ prit dès le début une option radicale en faveur du document authentique et plus particulièrement du document sonore, alors que

les méthodologues du CREDIF $^{2}$ tout en proposant d'aller vers le document authentique et de lui faire une place au niveau 2 recommandaient une certaine prudence. [...] Daniel Coste lui reprochait [...] de ne garantir ni une réception authentique par les apprenants ni une communication authentique dans la classe, 
ce qui lui paraissait plus important que l'authenticité des supports. (Cité par Boyer, Butzbach-Rivera \& Pendanx, 1990 : 31)

6 Les documents authentiques cités en exemple par les uns et les autres relèvent massivement des domaines iconiques et écrits :

Archipel, une des premières méthodes de l'approche communicative, favorise l'entrée des documents authentiques, essentiellement écrits et visuels, dans le matériel élaboré pour les débuts de l'apprentissage. (Cuq \& Gruca, 2017 : 403)

Une des caractéristiques de l'approche communicative est que l'enseignement recourt dans la mesure du possible à des documents dits authentiques [...]: par exemple un éditorial de journal, un calendrier, un menu de restaurant, un bulletin de nouvelles à la radio, etc. (Germain, 1993 : 207)

7 Les seuls documents oraux mentionnés sont les documents radiophoniques. On remarque l'absence totale d'échanges quotidiens dans la liste des documents authentiques. Et pour cause ! Ces interactions quotidiennes à la maison, dans la rue ou les commerces ne sont jamais enregistrées, contrairement aux documents radios ou télévisés. Ces discours oraux, fugaces par nature, ne sont pas aisément enregistrables et ne peuvent donc devenir des documents authentiques pour la classe. Parpette (2018) a analysé les raisons qui maintiennent les interactions orales quotidiennes dans le champ des discours fugaces non stabilisés par l'enregistrement, et qui, par voie de conséquence ne peuvent apparaître dans les documents authentiques, laissant ainsi toute la place aux documents radios.

8 Sur le plan méthodologique, parallèlement à l'introduction de documents authentiques iconiques et écrits dans les premiers niveaux (photos, publicités, annonces diverses, recettes, sites internet, etc.), et oraux à partir du niveau B1 et surtout B2 (essentiellement tirés d'émissions de radio et de télévision), se met en place une stratégie intermédiaire entre document fabriqué et document authentique. Face aux réticences de certains à l'égard de la notion d'authenticité, face également à la fréquente incompatibilité entre les documents authentiques et le niveau linguistique des premiers niveaux, apparaissent des documents, notamment oraux, qualifiés de « réalistes ». Il s'agit de se rapprocher le plus possible $\mathrm{du}$ discours d'origine tout en éliminant les difficultés qui pourraient contrecarrer le travail d'apprentissage :

Les documents oraux insérés dans les méthodes restent souvent fabriqués, réalisés avec des intentions pédagogiques, mais tentent de restituer l'authenticité du fonctionnement de l'oral hors contexte d'apprentissage ${ }^{3}$. (Cuq \& Gruca, $2017: 407$ )

9 Le champ du français sur objectif spécifique (FOS), tel que nous l'abordons depuis une quinzaine d'années (Mangiante \& Parpette 2004), remet à l'ordre du jour la réflexion sur les documents authentiques et notamment les documents authentiques oraux.

\section{FOS et documents authentiques}

10 Comme nous l'évoquions en introduction, le FOS confronte l'enseignant-concepteur à un domaine qui ne lui est pas familier voire inconnu. Il ne peut pas puiser dans son expérience personnelle pour imaginer les situations et discours des médecins en hôpital, des professionnels de la cuisine, ou des étudiants dans une université française. Cela exclut la stratégie des documents fabriqués fondés sur les connaissances du concepteur. Il suffit de s'être confronté une fois à l'élaboration de séquences de FOS pour mesurer la distance qui existe entre situations et discours tels qu'on les imagine et la réalité... Les étudiants de master de didactique duFLE en savent quelque chose! Cela explique 
pourquoi Mangiante et Parpette (2004:46-77) considèrent le travail de terrain et la collecte des données comme l'étape majeure de la démarche FOS.

Le FOS met donc au premier plan la problématique des documents authentiques. Le travail de terrain amène le concepteur à observer des lieux, des professionnels en action et leurs discours. Cela aboutit à des recueils de données rendant compte des situations de communication sous forme d'enregistrements vidéos ou audios, et d'écrits, auxquels peuvent être adjointes des séries de photographies. Citons quelques exemples de discours collectés :

- Programme FOS pour des médecins effectuant un séjour d'une année dans un hôpital français : consultation d'un patient, analyse d'un cas avec un médecin sénior, échange avec une infirmière à propos de la sortie d'un patient, demande de scanner par téléphone puis par écrit, etc. ;

- Programme pour des gardiens d'immeubles : échanges avec des occupants de l'immeuble à propos d'un dysfonctionnement, demande écrite d'intervention pour une réparation, commande de produits, échange pendant l'état des lieux d'un appartement et remplissage du formulaire, etc. ;

- Programme de français sur objectif universitaire (FOU) destiné aux étudiants en mobilité : cours magistraux, sujets et corrigés d'examen, relevés de notes, exposés d'étudiants, documents de lecture, etc.

Les documents authentiques en FOS n'échappent pas aux interrogations qui les ont accompagnés à l'origine pour le français général. Restent d'actualité en effet les questions de niveau de langue, de relation entre discours et contexte, et d'authenticité des situations une fois celles-ci transférées en classe. Mais le FOS y apporte des réponses spécifiques comme nous allons le voir.

\section{FOS, discours et contexte}

Le FOS met en évidence, de manière encore plus aiguë que le français général, le concept de «contexte» auquel la pragmatique attribue un rôle majeur dans l'interprétation des discours (Maingueneau, 2009: 33-36). Le contexte inclut les participants, le lieu, le moment et l'enjeu de la communication (contexte étroit). Mais il intègre également les arrière-plans culturels dans lesquels circulent les discours (contexte large). Selon les cas, ces arrière-plans peuvent désigner l'organisation de l'institution traitée (hôpital, université, entreprise), les relations entre les acteurs, les habitudes, etc.

Le travail de prospection sur des terrains inconnus du concepteur de FOS rend très visible le fait que nombre de discours ne prennent véritablement sens que par rapport à leur contexte d'utilisation. Le phénomène est certes largement identique dans les situations traitées par le français général, mais il est compensé, masqué en quelque sorte, par les connaissances partagées qui permettent aux apprenants d'aborder sans trop d'interrogation les contextes français mis en scène dans le matériel pédagogique. Le français général repose sur un fond commun de connaissances dans la mesure où il traite de la vie quotidienne : se présenter, se repérer, faire des achats, parler de son parcours scolaire ou professionnel, de ses goûts, de ses expériences, participer à un projet, suivre l'actualité, etc., ces sujets structurant quasiment toutes les méthodes de FLE jusqu'au niveau B1 inclus. Cette connaissance partagée est beaucoup moins à l'œuvre dans le champ du FOS qui traite de domaines professionnels, donc plus spécialisés et connus 
surtout des acteurs qui en font partie. Le traitement explicite du contexte devient donc indispensable.

En effet, ces discours oraux et écrits, cette part langagière du travail qui est le cœur de l'action de l'enseignant de langue, s'ils sont essentiels à la compréhension des situations et à la préparation des programmes de FOS, ne se donnent pas à lire aisément. Ils ne révèlent pas tout, comme l'illustre l'exemple suivant. Il s'agit des premières minutes d'une journée de classe de CE2. La maîtresse fait entrer les élèves dans la classe, et commence à échanger avec eux :

Fig. 1. - Classe de primaire.

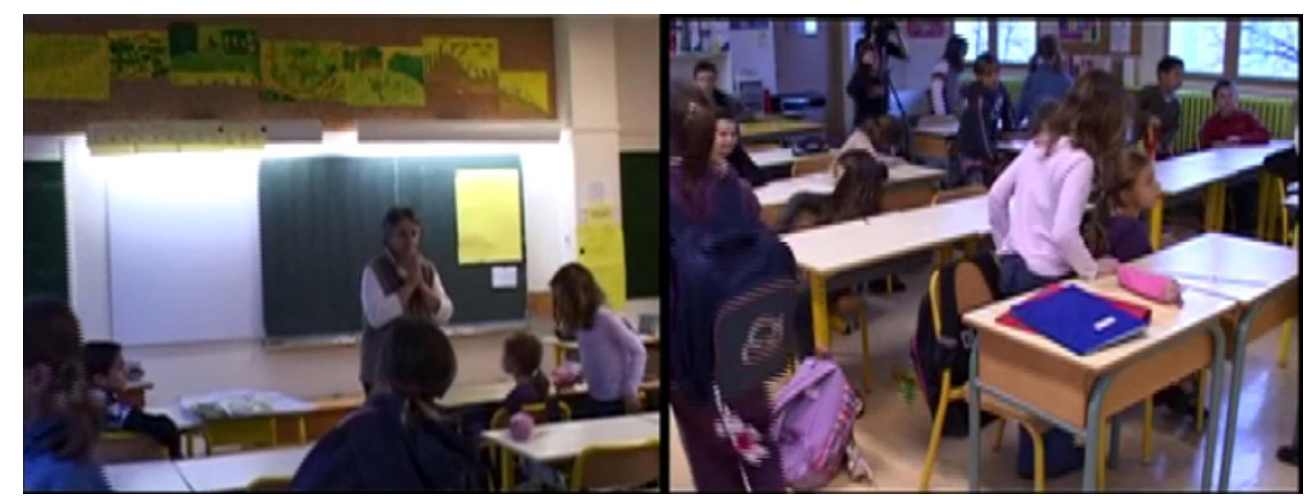

Extrait $^{4}$ de classe

$\mathrm{P}$ : on va essayer de démarrer rapidement / vous pensez à la cantine y a quelques autorisations // qui manquaient / c'est bon / on va commencer donc par / quelques / documents que je vous avais demandé de faire signer par vos parents / alors pour aller un petit peu vite / il y avait euh rappelez-moi

E1 : la poésie et

$\mathrm{P}:$ poésie non /

E2: mais non

E3 : non la fiche de lecture et l'évaluation d'histoire /

$\mathrm{P}$ : alors ce que je propose c'est que / Élise tu récupères les évaluations d'histoire s'il te plaît // les cahiers et puis euh / Lorena tu ramasses les cahiers pour la fiche de lecture / rapidement / pour ceux qui ont oublié on règlera ça en début d'après-midi // s'il y a des oublis /

Les passages indiqués en gras réfèrent à des fonctionnements de l'école évoqués de manière très allusive. Ainsi la phrase de l'enseignante "vous pensez à la cantine » provoque immédiatement la sortie et le retour rapide de plusieurs élèves. Cette consigne et ses effets interrogent tout observateur extérieur à l'école. Seule l'explication fournie par l'enseignante lors d'un entretien hors de la classe permet de donner du sens à cet échange, à savoir qu'au début de chaque journée, les élèves qui mangent à la cantine doivent inscrire leur nom sur une feuille affichée dans le couloir. De même, l'évocation des « autorisations qui manquaient » ne devient claire que si l'on sait qu'on ne peut filmer des enfants dans une école qu'avec l'accord des parents et que l'enseignante avait donc demandé aux parents une autorisation pour que le cours soit enregistré. Tout comme l'énoncé sur les documents à "faire signer par les parents » - dont on apprend quelques secondes plus tard qu'il s'agit d'une fiche de lecture et de l'évaluation d'histoire - ne prend son sens qu'à partir du moment où l'on connaît cette habitude scolaire, qui consiste à faire signer certains devoirs par les parents pour les informer du travail et des résultats de leurs enfants. On peut étendre cette analyse aux consignes «Élise tu récupères les évaluations d'histoire s'il te plait » et «Lorena tu ramasses les cahiers pour la fiche de 
lecture » dont la signification est claire en surface mais peut interroger qui ne connait cette habitude de l'école de confier aux élèves des tâches d'organisation de la classe. Pour mieux saisir ce lien entre les discours et leur ancrage culturel, on peut imaginer la surprise de parents français qui au Japon entendraient un enseignant demander à leurs jeunes enfants de prendre seau et balai... parce qu'au Japon, ce sont les élèves qui font le ménage de leur école.

\section{Ancrage culturel, extériorité et décontextualisation}

Cette relation entre discours et contexte, nécessaire au fonctionnement performant des situations de communication, met en situation d'extériorité tout individu confronté au domaine concerné alors qu'il n'en est pas un acteur habituel. Dans le cadre des formations FOS, cette extériorité se joue sur plusieurs plans.

Elle intervient tout d'abord au niveau global de l'organisation des institutions professionnelles ou universitaires qui ne sont pas construites, vécues, mises en discours de manière similaire d'un pays à l'autre. Le monde du travail obéit à des règlementations nationales. Les fonctions des infirmières, par exemple, sont différentes entre la France et d'autres pays. Certains actes techniques qui relèvent de leur activité en France ne leur sont pas autorisés en Grande-Bretagne ou en Chine, ceci étant essentiellement lié à la durée des formations. On trouve par exemple dans Le français des infirmiers la mention suivante :

L'électrocardiogramme est le seul examen technique prescrit que l'IDE ${ }^{5}$ est autorisé

(e) à pratiquer. Les autres formes d'investigation comme [...] sont des actes prescrits effectués par des manipulateurs [...] voire directement par des médecins. (Goy-Talavera, Gardette-Tria \& Perez, $2017:$ 183)

De même, l'interrogatoire d'un patient par le médecin dans un service d'urgence obéit à un protocole qui n'est pas universel. Faire des études supérieures dans un pays autre que le sien suppose également pour un étudiant allophone de connaître et comprendre l'organisation de l'institution universitaire d'accueil, les différences pouvant être importantes entre un système gratuit ou payant, sélectif ou ouvert, organisé suivant le système Licence-Master-Doctorat ou non, avec une organisation en semestres, des examens avec deux sessions, des modalités de travail et des relations enseignantsétudiants différentes, etc. La maîtrise du contexte est donc nécessaire pour comprendre les situations professionnelles et leurs discours. L'exemple vu plus haut relève d'un programme de FOS destiné à des parents migrants désireux de suivre la scolarité de leurs enfants. Le travail linguistique est organisé autour de la découverte du fonctionnement administratif et pédagogique de l'école. Les différentes séquences proposées concernent notamment la présentation des personnels de l'école et leurs fonctions respectives, les types de locaux, les horaires, les réunions de parents, le carnet de liaison, les comportements à adopter en cas d'absence de son enfant, etc. Ces réalités de l'école, on l'a vu, structurent fortement les discours qui y circulent, mais comme tout vécu partagé, elles le font de manière sous-jacente, très implicite. Face à cette réalité, on comprend que les parents-apprenants se sentent extérieurs à la situation, comme l'est un médecin cambodgien ou cubain confronté en formation à des scènes d'hôpital français.

L'extériorité est également liée au dispositif même du cours de langue. La classe de langue dans laquelle sont introduites les données tirées des situations professionnelles n'est pas le lieu d'émergence des discours étudiés. L'apprenant ne se trouve pas dans le temps et 
dans l'espace qui permettent de donner du sens à tous les énoncés. Les discours authentiques introduits en classe de FLE-FOS, sous forme d'enregistrements vidéos par exemple, sont montrés hors de leur contexte d'origine, ce qui peut créer de l'opacité et de l'incompréhension. On voit bien avec ce début de cours en primaire que le discours ne « commence " pas à $8 \mathrm{~h} 30$ ce matin-là. Il poursuit le discours et les actions de la veille, des jours et semaines qui ont précédé. L'extériorité est créée par la contrainte de tout cours de langue de travailler sur des extraits de situations et de discours, sur des moments isolés de ce qui les a précédés. On comprend les réticences de certains méthodologues soulignant que le recours aux documents authentiques ne garantit pas une réception authentique.

21 L'insertion de discours authentiques dans un programme de FOS s'accompagne, du fait de cette extériorité, d'un phénomène de décontextualisation qui peut générer de l'opacité, ce qui est bien évidemment l'inverse de ce qui est visé par la collecte des données authentiques. Le problème est particulièrement crucial pour les données orales, les interactions orales s'inscrivant plus fortement que les écrits dans la continuité temporelle, dans l'histoire conversationnelle entre les interlocuteurs.

Les concepteurs de FOS sont donc confrontés à une situation quelque peu paradoxale : les données authentiques, perçues en didactique comme des atouts parce qu'elles rapprochent le cours de FLE de la réalité des discours, constituent en même temps un facteur de complexification pour la compréhension des discours ciblés. Se pose donc la question du traitement de cette opacité potentielle liée à la décontextualisation des discours authentiques utilisés dans la classe de FOS.

\section{Stratégies de recontextualisation}

23 L'analyse de différents ouvrages pédagogique en FOS fait apparaître deux stratégies différentes pour restituer les discours authentiques dans leur contexte et leur rendre leur signification: la reconstitution, d'une part, et la création du tandem «données existantes-données sollicitées ", de l'autre.

$24-L a$ reconstitution: c'est la plus répandue. À partir du travail de terrain, observations et entretiens notamment, le concepteur, avec les acteurs du domaine concerné, organise des reconstitutions au plus près des situations réelles parce que ces reconstitutions sont réalisées par les professionnels eux-mêmes, dans les lieux mêmes où se déroule chaque situation de communication. Le français des médecins (Fassier \& Goy-Talavera, 2008) constitue un exemple marquant de cette approche. L'ouvrage est construit autour de 40 vidéos représentant les différentes situations de communication auxquelles sont confrontés les médecins étrangers en stage dans un hôpital français (arrivée sur le lieu de stage, contacts avec l'équipe administrative, situations de travail avec les patients, les médecins, les infirmiers, les différents services, présentation de travaux de recherche, etc.). Chaque protagoniste joue son propre rôle, à l'exception des patients pour des raisons déontologiques aisément compréhensibles. La même conception est à l'œuvre dans Le français des infirmiers (Goy-Talavera, Gardette-Tira \&Perez, 2017). 
Figure 2. - Le français des médecins (Fassier \& Goy-Talavera, 2008, vidéo 23).

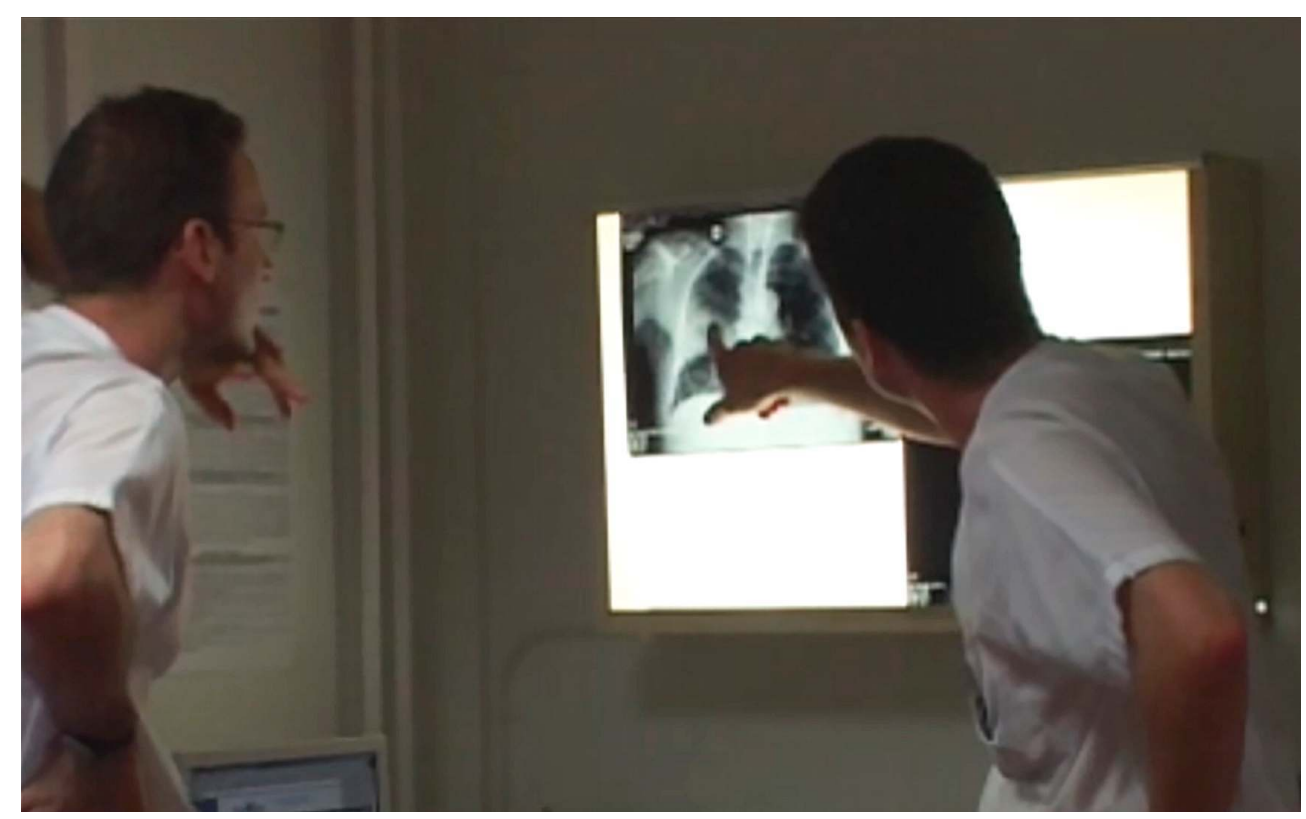

Pour éviter l'opacité créée par la décontextualisation, le contexte est réintroduit par une élucidation des implicites éventuels liés par exemple à la temporalité des situations : les échanges oraux-vidéos entre deux professionnels réfèrent à des faits présents dans les vidéos précédentes ; par ailleurs, les discours très allusifs sont évités. Les situations sont reconstituées sur le mode de l'autonomie discursive, qui en conditionne la compréhension par les apprenants allophones.

26 - La seconde stratégie, plus récente, est celle qui a été mise en place par la collection Français sur objectif universitaire (FOU) des PUG sur le modèle de la démarche FOS proposé par Mangiante et Parpette (2004). Elle consiste à combiner deux types de données :

- Les données existantes, c'est-à-dire celles qui sont naturellement présentes sur le terrain dans les situations visées, professionnelles ou universitaires : échanges oraux, documents écrits, iconiques, qui sont collectées telles qu'elles sont produites. Dans le cadre des formations FOU par exemple, il s'agit essentiellement, pour l'oral, des cours (cours magistraux et travaux dirigés), des exposés d'étudiants, et, pour l'écrit, des articles et ouvrages à lire, ainsi que des sujets d'examen et leurs corrigés.

- Les données sollicitées, c'est-à-dire les discours (interviews) provoqués par le concepteur de FOS-FOU qui interroge les professionnels pour les amener à expliciter les situations de communication traitées, et crée ainsi des documents authentiques oraux complémentaires.

27 C'est ainsi que, dans un programme destiné aux étudiants en mobilité, on retrouve combinés un extrait de cours magistral intitulé Grands auteurs en gestion avec une interview de l'enseignant expliquant l'objectif général du cours, son contenu et les modalités d'examen (Parpette \& Stauber, 2014). Les données sollicitées permettent de restituer le contexte d'une donnée authentique - un extrait de cours magistral d'une dizaine de minutes - qui ainsi isolé a perdu une large part de son contexte: à quel enseignement appartient-il ? en quelle année ? quelles compétences vise-t-il à développer chez les étudiants? etc. Les données sollicitées permettent également de restituer un contexte plus large : interviews d'enseignants décrivant l'ensemble d'un cursus de licence 
(Mangiante \& Raviez, 2015), témoignages d'étudiants expliquant les méthodes de travail dans leur discipline, etc. Ces différentes données constituent autant de supports de formation linguistique - sous forme de documents authentiques - pour les apprenants dans les programmes de FOS, comme on peut le voir dans les ouvrages évoqués.

Figure 3. - Cours magistral d'économie-gestion $1^{\mathrm{re}}$ année : Les grands auteurs en gestion (discours existant).

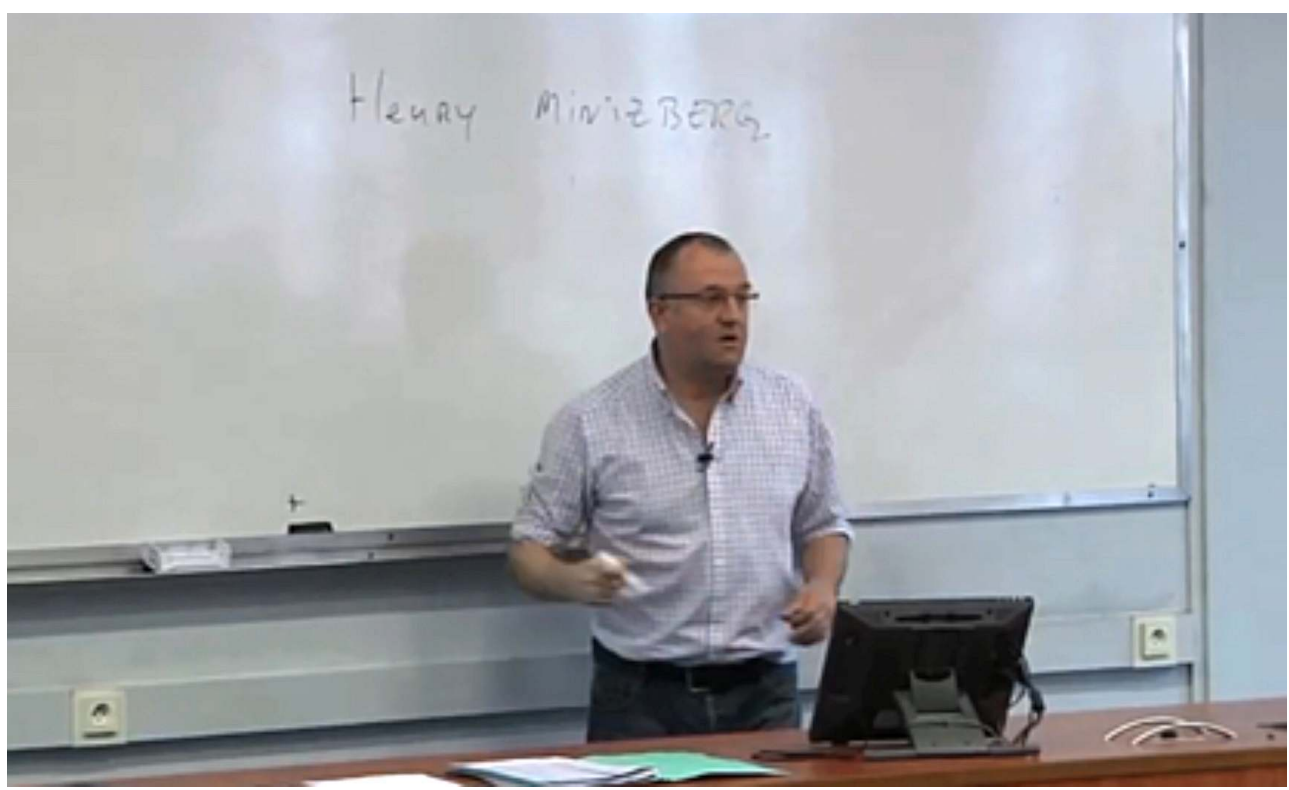

Figure 4. - Interview sur le cours Les grands auteurs en gestion (discours sollicité).

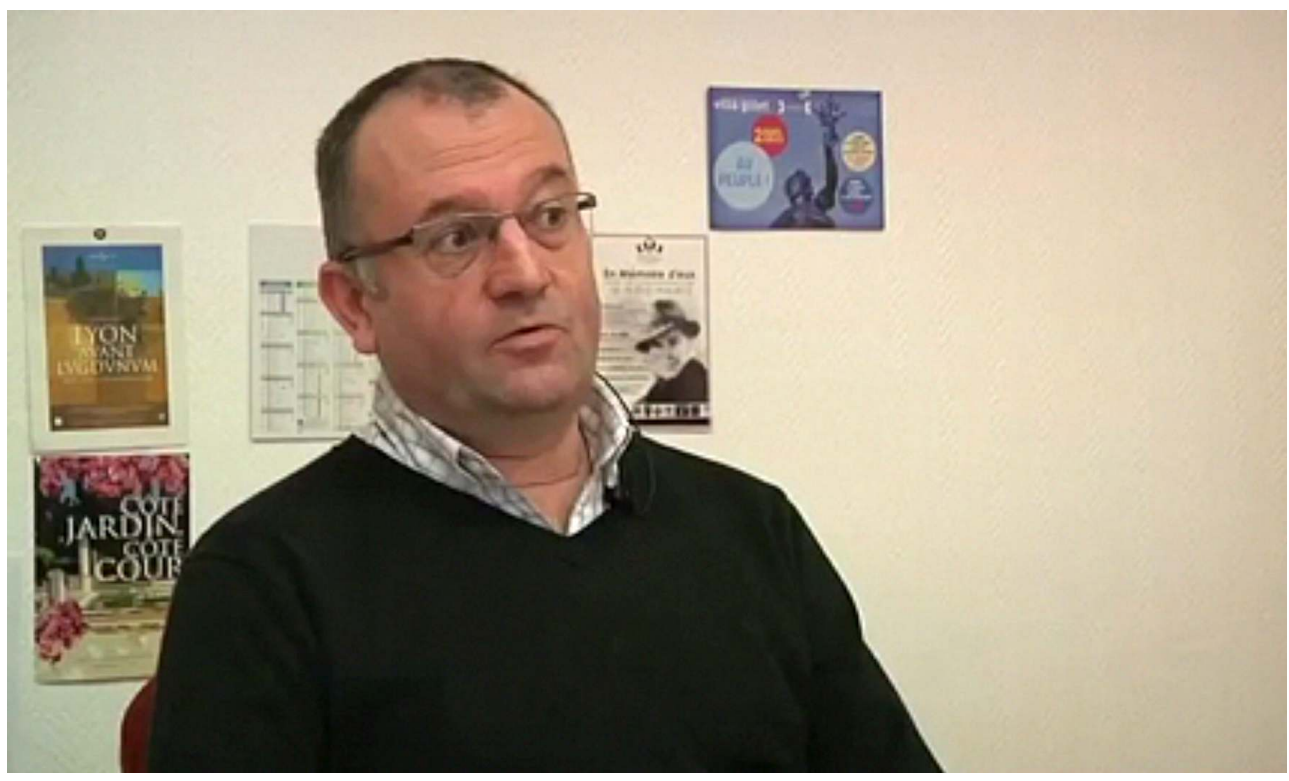

Cette seconde stratégie " données existantes + données sollicitées » a été reprise sous une forme différente par l'ouvrage Le français des infirmiers (Goy-Talavera, Gardette-Tira \& $\mathrm{P}$ erez, 2017 : 94). Les informations contextuelles sollicitées auprès des gens du terrain pour élucider l'arrière-plan culturel n'ont pas abouti à des interviews, mais à des écrits d'accompagnement, servant d'introductions aux séquences, par exemple celui-ci dans la séquence Accueillir un patient : 


\section{Informer les proches}

L'IDE ${ }^{6}$ est régulièrement en contact avec les proches des patients, soit lorsqu'il/elle les rencontre à l'hôpital, soit par téléphone. Informer la personne de confiance relève du rôle de l'IDE, mais les informations qu'il lui est possible de donner sont très limitées. C'est en effet au médecin et non à l'IDE de parler des problèmes, par exemple de donner des résultats d'examen qui ne sont pas bons, etc.

Ce document est destiné à apporter des précisions éclairant les fonctions de l'IDE, lequel ne peut délivrer qu'un certain nombre d'informations aux familles des patients. La forme est certes différente, mais la combinaison est bien là, entre discours in situ et restitution de l'arrière-plan contextuel.

\section{Changement de perspective}

Le français sur objectif spécifique s'assigne deux fonctions dans sa démarche de formation : le développement des compétences linguistiques requises par le milieu ciblé, d'une part, et l'information sur le contexte, de l'autre. Si ces deux éléments ne sont pas totalement séparés - toute séquence pédagogique portant à travers les documents traités en compréhension une information sur le contexte-, le FOS en fait cependant deux composantes distinctes avec des modalités de combinaison qui peuvent varier selon les situations. Si dans les formations FOS qui ont lieu en France, les deux types de données sont traités de manière étroitement combinées, d'autres cas de figure peuvent amener à les traiter différemment.

31 Ainsi, pour les formations qui ont lieu dans le pays d'origine, il est tout à fait envisageable, parallèlement au travail en français sur les compétences langagières, de traiter l'information sur le contexte large (organisation de l'hôpital, de l'université, etc.) en langue maternelle. C'est ce que propose le site Étudier en francophonie créé par l'AUF7 au Brésil pour les étudiants visant une mobilité en pays francophone: il comporte des séquences de formation linguistique en français (compréhension de vidéos sur la vie des étudiants, les activités sur les campus, les formes de travail à l'université, etc.) parallèlement à des informations sur les systèmes universitaires du Québec, de la Belgique ou de la France qui, elles, sont en portugais. Autre exemple: lorsque les formations FOS se déroulent en deux temps, d'abord dans le pays d'origine avant le départ, et ensuite en France, cette répartition entre les deux composantes apparait également, mais cette fois sur le plan chronologique. En effet, avant le départ, les étudiants s'intéressent plutôt à la connaissance du contexte et au fonctionnement des institutions qu'ils doivent rejoindre ; une fois sur place, leur intérêt s'oriente davantage vers la formation linguistique. Ces deux composantes se distinguent également par leurs modalités de collecte des données. Il est plus facile d'organiser des interviews (même à distance) que de collecter des discours existants in situ ${ }^{8}$.

Le FOS fait ainsi évoluer le périmètre du « document authentique ». Ce qui était nommé depuis les années 1970 " document authentique » correspond aux "discours existants » du FOS, ceux qui peuvent être captés ou collectés in situ. La notion d'authentique a été élargie à la connaissance du contexte, laquelle permet de donner leur pleine signification aux discours existants. Cette évolution peut être représentée sous la forme suivante : 
Figure 5. - Évolution de la notion d'authentique.

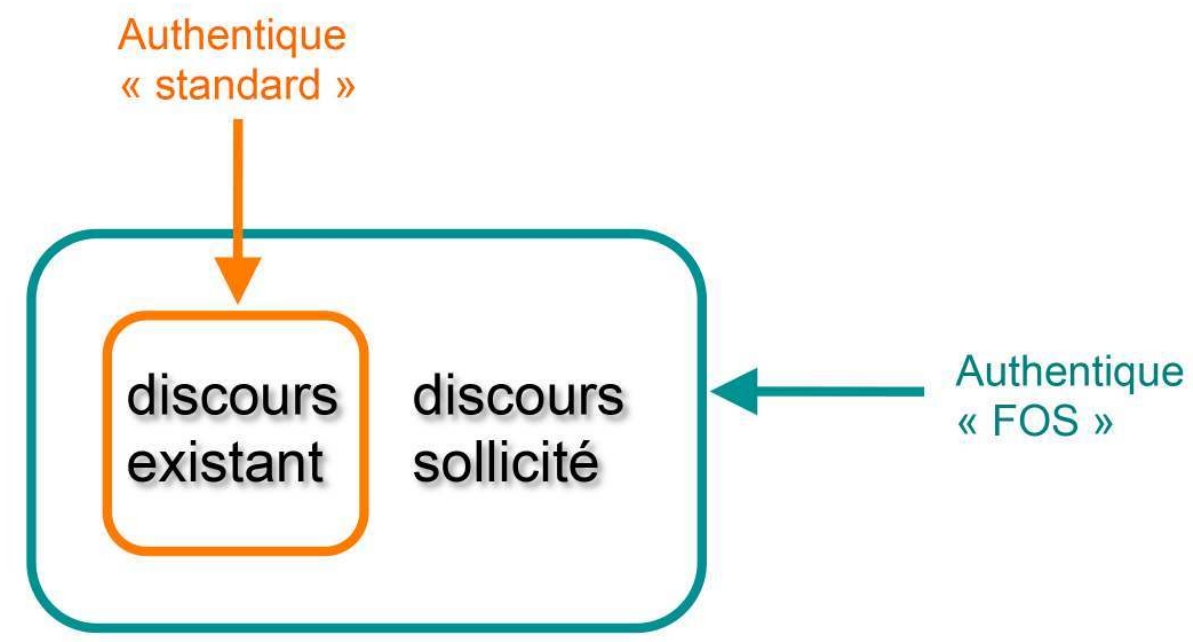

Cette conception des "données» authentiques (terme que l'on préfèrera à celui de « document » parce que plus global et intégratif) combine donc les discours EN situation avec les discours SUR les situations, un peu à la manière du travail du journaliste qui d'une part recueille les faits sur le vif, en filmant une action, puis va tendre son micro aux gens présents pour recueillir leur témoignage afin de (faire) comprendre l'événement. Les deux types de discours, devenus documents authentiques, sont intégrés conjointement dans les programmes de FOS.

\section{Conclusion}

La notion d'authentique concerne autant le français général que le français sur objectif spécifique, mais elle s'impose plus nettement dans le second dans la mesure où il touche à des domaines plus particuliers, moins connus de tous. La nécessité pour les apprenants allophones de se familiariser avec la dimension institutionnelle, voire historique, des situations traitées (le milieu hospitalier, l'école, le monde de la restauration, etc.) modifie la notion d'authentique. Initialement rattachée aux seuls « documents » présents dans les situations naturelles, elle s'élargit explicitement, dans la démarche FOS, via les discours sollicités, aux contextes d'ancrage des discours collectés dans les milieux ciblés. Elle apporte ainsi une réponse méthodologique aux interrogations des débuts de l'approche communicative.

\section{BIBLIOGRAPHIE}

ABE Danielle, CARTon Francis, CEMbalo Michel \& RÉGENT Odile (1979), « Didactique et authentique : du document à la pédagogie », Mélanges pédagogiques, Nancy : Université de Nancy. 
BOYer Henri, ButzBach-Rivera Michèle \& PendanX Michèle (1990), Nouvelle introduction à la didactique du français langue étrangère, Paris : CLE International.

CuQ Jean-Pierre (dir.) (2003), Dictionnaire de didactique du français langue étrangère, Paris : CLE International.

CUQ Jean-Pierre \& GRUCA Isabelle (2017), Cours de didactique du français langue étrangère ( $2^{\mathrm{e}}$ éd.), Grenoble : PUG.

COURTILLON Janine \& RAILlARD Sabine (1988), Archipel 1, Paris : Didier FLE.

DEBYSER Francis (1979), « La découverte du parlé grâce au document sonore ", Le français dans le monde, 145, 80-81.

FASSIER Thomas \& Goy-TALAVERA Solange (2008), Le français des médecins, Grenoble : PUG.

GERMAIN Claude (1993), Évolution de l'enseignement des langues : 5000 ans d'histoire, Paris : CLE International.

Goy-TalaVera Solange, Gardette-Tira Nathalie \& Perez Chrystel (2017), Le français des infirmiers, Grenoble : PUG.

MAINGUENEAU Dominique (2009), Les termes clés de l'analyse du discours, Paris : Seuil.

MANGIANTE Jean-Marc \& PARPETTE Chantal (2004), Français sur objectif spécifique : de l'analyse des besoins à l'élaboration d'un cours, Paris : Hachette.

MANGIANTE Jean-Marc \& PARPETTE Chantal (2011), Français sur objectif universitaire, Grenoble : PUG.

MANGIANTE Jean-Marc \& RAVIEZ François (2015), Réussir ses études de littérature en français, Grenoble : PUG.

Montredon Jacques, Calbris Geneviève, Cesco Claire, Dragoje Denise, Gschwind-Holtzer Gisèle \& LAVENNE Christian (1976), C'est le printemps, Paris : CLE International.

PARPETTE Chantal (2014), « Contexte local et stratégie de programmation en français sur objectif universitaire », I. Franic \& M. Ruet, Le français sur objectif universitaire : du concept à la pratique, Zagreb : FF Press, 11-23.

PARPETTE Chantal (2018, sous presse), «Quelle relation entre discours oral naturel et document oral authentique en FLE? ", Action didactique, 1, Université de Bejaia.

PARPETTE Chantal \& STAUBER Julie (2014), Réussir ses études d'économie-gestion en français, Grenoble : PUG.

\section{NOTES}

1. Bureau pour l'enseignement de la langue et de la civilisation françaises à l'étranger, né en 1967 de la fusion du BEL (Bureau d'études et de liaison pour l'enseignement du français dans le monde) et du CREC (Centre de recherche pour l'enseignement de la civilisation).

2. Centre de recherche pour la diffusion du français, créé en 1959.

3. Souligné par nous.

4. Conventions de transcription : / pause brève, // pause longue, $\uparrow$ intonation montante.

$\mathrm{P}=$ professeur $-\mathrm{E}=$ élève.

5. IDE : infirmier(ère) diplômé(e) d'État.

6. Idem. 
7. Agence universitaire de la francophonie

8. Sur la diversité des programmes en FOU en fonction des contextes, voir Parpette (2014).

\section{RÉSUMÉS}

L'élaboration des programmes de français sur objectif spécifique fait largement appel aux données authentiques recueillies lors du travail de terrain mené par le concepteur. Mais les "documents authentiques", largement valorisés dans l'approche communicative depuis près de 40 ans ne rendent pas toujours les services attendus. Tirés de leur contexte d'origine, et donc coupés des arrière-plans culturels qui les sous-tendent, ils perdent souvent une bonne partie de leur signification. Cela a conduit la didactique du FOS à proposer de nouvelles stratégies d'utilisation des données de terrain et à redéfinir la notion de document authentique.

The development of French language programs with a specific objective makes extensive use of authentic data collected during the fieldwork conducted by the researcher. However, the "authentic documents", widely valued in the communicative approach for nearly 40 years, do not always render the services expected. Drawn from their original context and thus cut off their cultural backgrounds that underlie them, they often lose part of their significance. This has led didactics of French with a specific objective to offer new strategies in order to use these field data accordingly and to redefine the notion of authentic documents.

\section{INDEX}

Keywords : French with a specific objective, authentic document, context, existing speech, solicited speech

Mots-clés : français sur objectif spécifique, document authentique, contexte, discours existant, discours sollicité

\section{AUTEURS}

\section{SOPHIE DUFOUR}

Université Paul-Valéry Montpellier 3, laboratoire Praxiling

CHANTAL PARPETTE

Université de Lyon, laboratoire ICAR 